\title{
UCRL-CONF-221583
}

LA W REN CE LIVERM ORE NATIONAL LABORATORY
Electron Beam-induced Light Emission and Transport in GaN Nanowires

J. W. Tringe, W. J. MoberlyChan, C. G. Stevens, A. V. Davydov, A. Motayed

May 23, 2006

1st International Conference on Nano-Networks Lausanne, Switzerland September 14, 2006 through September 16, 2006 
This document was prepared as an account of work sponsored by an agency of the United States Government. Neither the United States Government nor the University of California nor any of their employees, makes any warranty, express or implied, or assumes any legal liability or responsibility for the accuracy, completeness, or usefulness of any information, apparatus, product, or process disclosed, or represents that its use would not infringe privately owned rights. Reference herein to any specific commercial product, process, or service by trade name, trademark, manufacturer, or otherwise, does not necessarily constitute or imply its endorsement, recommendation, or favoring by the United States Government or the University of California. The views and opinions of authors expressed herein do not necessarily state or reflect those of the United States Government or the University of California, and shall not be used for advertising or product endorsement purposes. 


\section{Electron Beam-induced Light Emission and Transport in GaN Nanowires}

\author{
Joseph Tringe, Warren MoberlyChan, \\ and Charles Stevens \\ Chemistry and Materials Science Directorate \\ Lawrence LivermoreNational Laboratory \\ Livermore, California, USA
}

\author{
Albert Davydov and Abhishek Motayed \\ Metallurgy Division, Material Science \& Engineering Lab \\ National Institute of Standards and Technology \\ Gaithersburg, Maryland, USA
}

\begin{abstract}
We report observations of electron beam-induced light from GaN nanowires grown by chemical vapor deposition. GaN nanowires were modified in-situ with deposited opaque platinum coatings to estimate the extent to which light is channeled to the ends of nanowires. Some evidence of light channeling was found, but wire microstructure and defects play an important role in light scattering and transport, limiting the extent to which light is confined.
\end{abstract}

Keywords-nanowire, GaN, light, waveguide, optical

\section{INTRODUCTION}

Optical interconnects are powerful components presently applied for high bandwidth communications among highperformance processors.[1-4] Future circuits based on nanometer-scale components could similarly benefit from optical information transfer among processing blocks. Strong light channeling (and even lasing) has been observed in GaN nanowires, suggesting that these structures could be useful building blocks in a future networked electro-optical processor.[5] However, the extent to which defects and microstructure control optical performance in nanowire waveguides has not been measured. In this study, we use electron microscopy and in-situ modification of individual nanowires to begin to correlate wire structure with light transport efficiency through GaN nanowires tens of microns long.

\section{EXPERIMENTAL METHODS}

$\mathrm{GaN}$ nanowires were grown in a horizontal furnace by reacting Ga vapor with ammonia at $850-900{ }^{\circ} \mathrm{C}$ as described elsewhere.[6] Ammonia was flowed at 50-100 sccm for 3-4 hours, resulting in a matrix of GaN nanowires and platelets on the nitride boat and quartz furnace liner. Typical nanowire dimensions are $20 \mu \mathrm{m}$ to $100 \mu \mathrm{m}$ in length and $100 \mathrm{~nm}$ to 400 $\mathrm{nm}$ in diameter. The entire matrix was harvested, dissolved in isopropanol and dispersed on single-crystal silicon substrates for subsequent imaging and modification. A duel-beam FEI 620 focused ion beam/scanning electron microscope (FIB/SEM) was used for imaging, cathodoluminescence (optical signal) characterization, and for deposition of opaque Pt pads at the ends of the nanowires, where light emission was expected to be concentrated because of channeling effects.[5] Light was excited in the nanowires by $10 \mathrm{kV}$ electrons with a nominal beam current of $15 \pm 3 \mathrm{nA}$. Electron-induced optical signals were collected with a $5 \mathrm{~mm}$ collimating lens positioned $\sim 20 \mathrm{~mm}$ from the sample during excitation, and transported out of the SEM vacuum chamber to a cooled spectrometer with UV-visible optical fibers. Background-subtracted signal was integrated for $60 \mathrm{~s}$ during every measurement.

As described elsewhere[7], GaN platelets are the source of GaN nanowires, and are typically 0.5 to $5 \mu \mathrm{m}$ in diameter with the normal of the largest face being parallel to the c-axis ([0001]-type direction) of the wurtzite structure. During growth, nanowires extend from the sides of the platelets such that the growth direction is parallel to the wurtzite a-planes (Fig. 1).
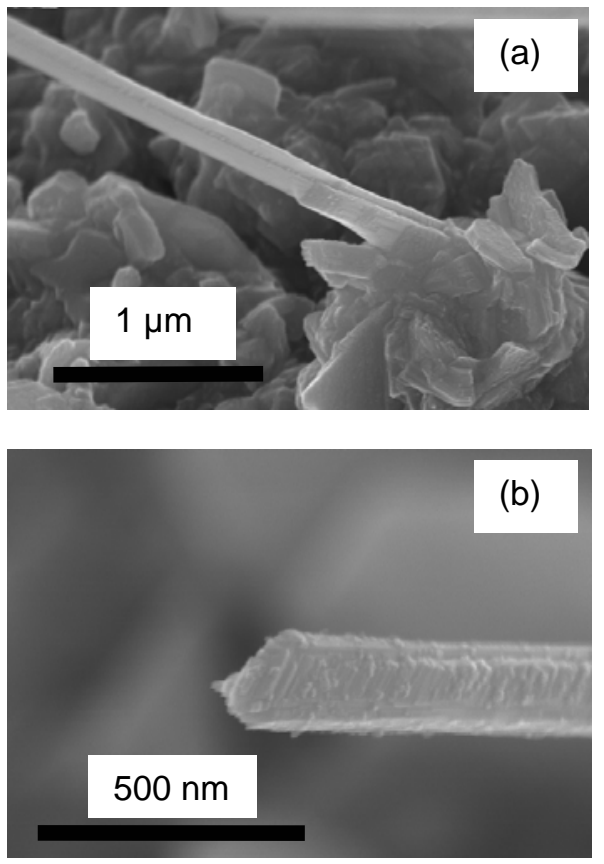

Fig. 1(a) GaN nanowire root attached to matrix (flake) (b) Nanowire tip; growth defects along with the extended structural defects as found in transmission electron microscopy (not shown) may cause light scattering in nanowires. 
Upon harvesting, however, nanowires typically break free of the generating platelets. The orientation of both platelets and wires was established by transmission electron microscopy and electron back-scattered diffraction patterns. The thinnest nanowires (<100 nm diameter) are often single crystals, but larger-diameter wires are typically composed of groups of parallel crystals, all with growth directions parallel to the wurtzite a-planes. Polycrystalline nanowires were examined for this study because of their higher absolute levels of light emission.

\section{RESUlts}

A typical GaN nanowire, $40 \mu \mathrm{m}$ long and 150-200 nm in diameter, was harvested and placed on a Si substrate as shown in Fig. 2(a). Note the presence of several small GaN platelets and multi-platelet structures also dispersed on the substrate. Cathodoluminescence signals were obtained from wire regions $400 \mathrm{~nm}$ wide by $2 \mu \mathrm{m}$ long, shown schematically in Fig. 2(a). An area larger than the width of the nanowire was selected for measurement to prevent charge accumulation in the wire, and to avoid problems associated with beam drift.
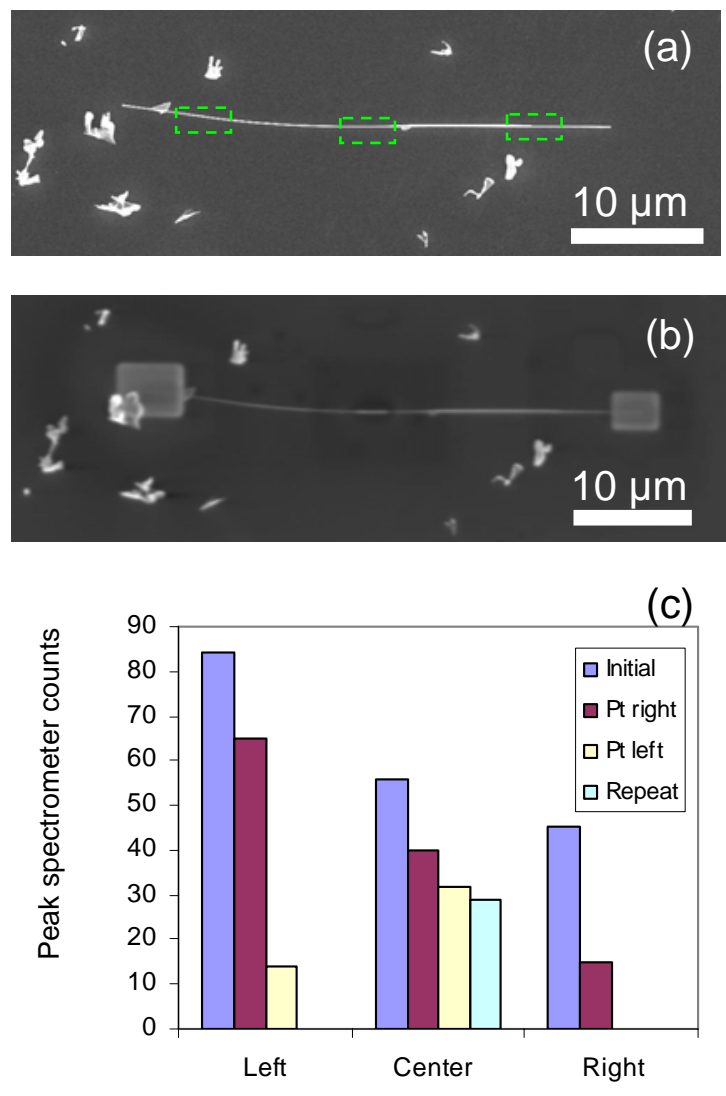

Fig. 2(a) 150-200 nm diameter nanowire before Pt deposition, (b) after Pt deposition, and (c) GaN band gap peak signal at locations shown in (a).

Optical signals were confirmed spectroscopically to have been generated in $\mathrm{GaN}$, and background signals from Si substrate and all other sources were measured to be $<10 \%$ of the typical
GaN signal. After initial survey of light output from the nanowire excited at various locations, an opaque Pt pad at least $200 \mathrm{~nm}$ thick was deposited on the right side of the nanowire by local electron-beam induced decomposition of the Pt precursor (Trimethyl)methylcyclopentadienlylplatinum. A second opaque Pt pad was deposited on the left. The final structure is shown in Fig. 2(b). The magnitude of the light emission peak associated with the GaN band gap is shown in Fig. 2(c), before and after Pt deposition.

A second nanowire, 300-400 nm in diameter, is shown in Fig. 3(a) before Pt deposition. Fig. 3(b) shows the wire after Pt pad deposition. Cathodoluminescence signals were obtained from areas $2 \mu \mathrm{m}$ by $500 \mathrm{~nm}$. The magnitude of the GaN band gap-associated optical signal before and after Pt deposition is shown in Fig. 3(c).
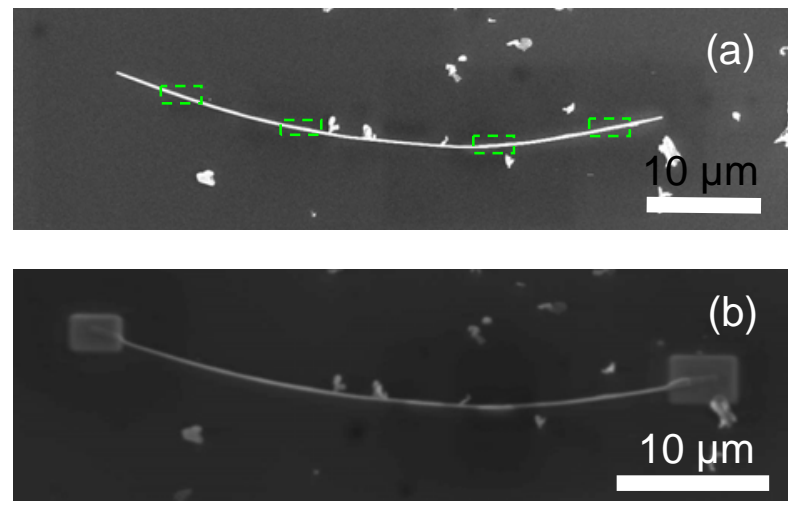

(c)

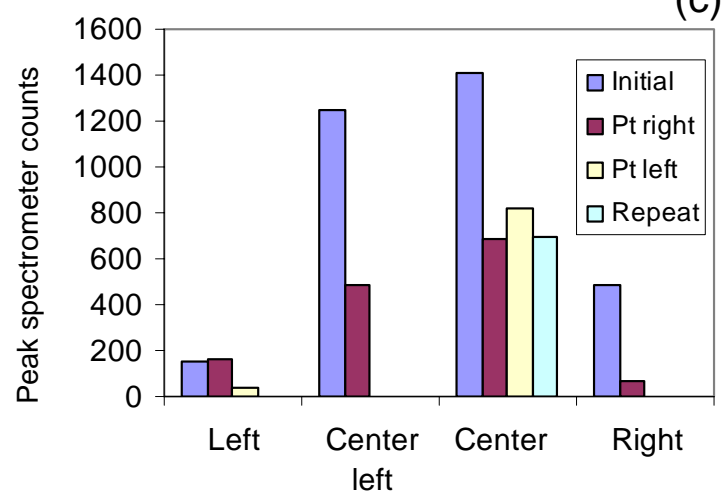

Fig. 3(a) 300-400 nm diameter nanowire before Pt deposition, (b) after Pt deposition, and (c) GaN band gap peak signal at locations shown in (a).

\section{DISCUSSION}

Both nanowires exhibited reductions in light intensity following deposition of Pt on one end, disproportionate to the area covered and in areas far away from the deposition location. This is consistent with light channeling, as previously observed in GaN by near-field scanning optical microscopy [5]. However, especially for the 150-200 nm diameter wire, reductions in light intensity were modest. Note 
that even without Pt deposition, the same location measured consecutively typically yielded integrated peak values $10-15 \%$ lower the second time, possibly due to migration and accumulation of carbon on the GaN nanowires during the long (60 s) integration time necessary for adequate signal to noise levels to be achieved.

Reductions in light intensity observed after right side Pt deposition at the center left and center positions of the 300$400 \mathrm{~nm}$ diameter wire, and at the left position of the 150-200 $\mathrm{nm}$ diameter wire, suggest that some channeled light is being blocked by the deposited Pt. However, when both ends are blocked, light from the center-excited wire does not drop to near-background levels, indicating some light is being emitted all along the length of the wire. Light scattering along the length of the wire is likely facilitated by the presence of extended defects such as stacking faults and surface steps along the sides of the nanowires. Previously, low-defect nanowires have been observed to be frequently oriented with the long direction parallel to [0001]. However, among [0110]oriented wires, internal defects and surface steps are common [8]. Since our wires were primarily [0110]-oriented, it is not unexpected that extended defects are present as observed by SEM (Fig. 1), and each of these defects can act as a scattering center. It is interesting to note that the light intensity from center-excited wires drops most when the side which was initially brightest is covered, indicating that light generated in the wires may have been preferentially channeled to this end. Light may be blocked at the other end by defects or the presence of a residual metallic precursor.

Dramatic reductions in light intensity at the ends of the nanowires following Pt deposition on those same ends may result from light being blocked by Pt outside of the target deposition area. Note, however, that $10 \mathrm{keV}$ electrons readily traverse $200 \mathrm{~nm}$-thick $\mathrm{Pt}$ : the range for $10 \mathrm{keV}$ electrons in $\mathrm{Pt}$ is estimated to $370 \mathrm{~nm}$ in the continuous slowing-down approximation[9]. Therefore, even if the beam is focused on a Pt-covered area, light will still be generated. However, this light will be readily absorbed by the Pt. Using Lambert's law with an absorption coefficient of $2.3 \times 10^{5} \mathrm{~cm}^{-1}$ for $3.4 \mathrm{eV}$ light (appropriate for light generated from the GaN band gap), we calculate that a $200 \mathrm{~nm}$-thick Pt layer is sufficient to reduce light intensity by a factor of $\sim 2 \times 10^{8}$. Although no Pt was observed on the wires outside of the target area, the effect on optical signals of Pt deposition outside of the target regions cannot be excluded and may even affect the center areas. Since similar thicknesses of Pt were deposited on left and right sides of the wires, however, and since the effects on wire light emission of outside-target Pt coverage is likely cumulative, it is reasonable to expect that center-excited light would drop similarly after Pt deposition on right and left sides. Especially for the 300-400 nm wire, however, this is not observed. Instead, light drops significantly following Pt deposition on the right side, and even increases slightly following Pt deposition on the left side, again suggesting a degree of light channeling.

\section{SUMMARY}

Light emission observed from Pt-modified GaN wires, 200$400 \mathrm{~nm}$ in diameter, is consistent with light channeling to the ends, though some light emission is found to occur all along the length of the nanowires. Extended defects such as stacking faults and surface steps likely cause significant scattering which limits the performance of these largediameter nanowires as waveguides. Smaller nanowires with fewer defects are presently under investigation for comparison.

\section{ACKNOWLEDGMENT}

The authors thank Dr. Maoqi He at Howard University for providing nanowire samples. This work was performed under the auspices of the US Dept. of Energy by the Univ. of California Lawrence Livermore National Laboratory under Contract W-7405-ENG-48.

\section{REFERENCES}

[1] D. M. Taylor, C. R. Bennett, T. J. Shepherd, L. F. Michaille, M. D. Nielsen, and H. R. Simonsen, "Demonstration of multi-core photonic crystal fibre in an optical interconnect," Electronics Letters, vol. 42, pp. 331332, 2006.

[2] T. Yamashita and C. J. Summers, "Evaluation of selfcollimated beams in photonic crystals for optical interconnect," IEEE Journal on Selected Areas in Communications, vol. 23, pp. 1341-1347, 2005.

[3] A. L. Glebov, J. Roman, M. G. Lee, and K. Yokouchi, "Optical interconnect modules with fully integrated reflector mirrors," IEEE Photonics Technology Letters, vol. 17, pp. 1540-1542, 2005.

[4] A. C. Walker, S. J. Fancey, M. P. Y. Desmulliez, M. G. Forbes, J. J. Casswell, G. S. Buller, M. R. Taghizadeh, J. A. B. Dines, C. R. Stanley, G. Pennelli, A. R. Boyd, J. L. Pearson, P. Horan, D. Byrne, J. Hegarty, S. Eitel, H. P. Gauggel, K. H. Gulden, A. Gauthier, P. Benabes, J. L. Gutzwiller, M. Goetz, and J. Oksman, "Operation of an optoelectronic crossbar switch containing a terabit-persecond free-space optical interconnect," IEEE Journal of Quantum Electronics, vol. 41, pp. 1024-1036, 2005.

[5] J. C. Johnson, H. J. Choi, K. P. Knutsen, R. D. Schaller, P. D. Yang, and R. J. Saykally, "Single gallium nitride nanowire lasers," Nature Materials, vol. 1, pp. 106-110, 2002.

[6] M. Q. He, I. Minus, P. Z. Zhou, S. N. Mohammed, J. B. Halpern, R. Jacobs, W. L. Sarney, L. Salamanca-Riba, and R. D. Vispute, "Growth of large-scale GaN nanowires and tubes by direct reaction of Ga with NH3," Applied Physics Letters, vol. 77, pp. 3731-3733, 2000.

[7] A. Motayed, A. V. Davydov, M. D. Vaudin, I. Levin, J. Melngailis, and S. N. Mohammad, "Fabrication of GaNbased nanoscale device structures utilizing focused ion beam induced Pt deposition," Journal of Applied Physics, in press.

[8] W. J. MoberlyChan, G. Seryogin, I. Shalish, C.-Y. Wen, S.F. Hu, X.-J. Guo, V. Narayanamurti, and F. Spaepen, "Imaging defects in nanometer-scale semiconductor crystals," presented at Microsc. Microanal., 2005.

[9] NIST, ESTAR http://physics.nist.gov/PhysRefData/Star/Text/, 2006. 